\title{
DIAGNÓSTICO DE MORTE ENCEFÁLICA: NOSSOS MÉDICOS ESTÃO PREPARADOS?
}

\author{
DIAGNOSIS OF BRAIN DEATH: ARE OUR DOCTORS PREPARED?
}

\author{
Abraão Oliveira Tavares ${ }^{1}$ \\ Paulo Antônio Farias Lucena² \\ Luciana Modesto de Brito $^{3}$ \\ Ankilma do Nascimento Andrade Feitosa ${ }^{4}$
}

RESUMO: OBJETIVO: Avaliar o conhecimento dos médicos de UTI (unidade de terapia intensiva) sobre o diagnóstico de morte encefálica. METODOLOGIA: Tratase de estudo transversal e quantitativo realizado por levantamento de dados a partir de um questionário. O questionário foi aplicado a médicos que trabalham na UTI do Hospital Regional de Cajazeiras e Hospital Regional de Sousa. O procedimento de coleta de dados foi executado através de dados primários, pré-determinados pelo pesquisador, por meio de questionário enviado via link para acesso ao formulário, utilizando ferramenta de WhatsApp, o qual foi destinado aos médicos plantonistas das referidas UTIs. RESULTADOS: Dos 16 médicos contatados, apenas oito profissionais se disponibilizaram a responder, os demais não justificaram o desejo de não participar. No cenário das UTIs do interior paraibano, o perfil dos médicos plantonistas é caracterizado, em sua maioria, com menos de 10 anos de exercício da profissão e sem especialidade médica. Apenas dois médicos (25\%) apresentavam residência médica em medicina intensiva. A resposta dos médicos participantes quanto à capacidade técnica para diagnosticar ME foi de acordo com o esperado, 62,5\% não estavam aptos a determinar ME. Um dos entraves encontrados é o fato que $75 \%$ dos médicos nunca receberam proposta para participar da capacitação e 50\% desconhecem que o CFM ofereça a capacitação para determinar morte encefálica. As respostas ao questionário também evidenciaram que apenas metade dos médicos que realizaram abertura de protocolo concluíram o diagnóstico, mas também há um médico que, sem capacitação, declarou ter fechado diagnóstico de ME. CONCLUSÃO: A Resolução CFM $n^{\circ}$ 2.173/2017 aumenta a credibilidade e confiança na medicina brasileira, traz atualização de conhecimentos de um diagnóstico desafiador e que tem impactos

\footnotetext{
${ }^{1}$ Acadêmico do curso de Medicina da Faculdade Santa Maria, Cajazeiras, Paraíba, Brasil.

2 Médico, Mestre, docente da Faculdade Santa Maria, Cajazeiras, Paraíba, Brasil.

3 Médica, docente do curso de Graduação em Medicina da Faculdade Santa Maria, Cajazeiras, Paraíba Brasil.

${ }^{4}$ Enfermeira, Doutora, docente da Faculdade Santa Maria, Cajazeiras, Paraíba, Brasil.
} 
indiretos na esperança de salvar mais vidas. Após concluir a análise deste estudo, evidencia-se a baixa habilitação para diagnóstico de ME portada pelos médicos diante de um cenário de UTI que apresenta significativos óbitos por morte encefálica. A destreza na determinação de ME é imprescindível para a doação de órgãos, por isso que merece sua devida relevância. Diante do apresentado, esperase que este estudo instigue o CFM a oferecer mais oportunidades de capacitação, e que os profissionais médicos busquem um melhor aperfeiçoamento e atualização dos seus conhecimentos para determinar morte encefálica de acordo com as novas normas.

Palavras-chave: Morte encefálica; Diagnóstico; Unidades de Terapia Intensiva.

ABSTRACT: OBJECTIVE: To evaluate the knowledge of ICU doctors (intensive care unit) about the diagnosis of brain death. METHODOLOGY: This is a cross-sectional and quantitative study carried out by collecting data from a questionnaire. The questionnaire was applied to doctors working in the ICU of the Hospital Regional de Cajazeiras and Hospital Regional de Sousa. The data collection procedure was performed using primary data, pre-determined by the researcher, through a questionnaire sent via link to access the form, using a WhatsApp tool, which was intended for on-duty doctors at the referred ICUS. RESULTS: Of the 16 doctors contacted, only eight professionals were available to answer, the others did not justify the desire to not participate. In the scenario of ICUs in the interior of Paraiba, the profile of doctors on duty is characterized, in most cases, with less than 10 years of practice and without medical specialty. Only two doctors (25\%) had medical residency in intensive care. The response of participating physicians regarding the technical capacity to diagnose BD was in line with expectations, $62.5 \%$ were not able to determine BD. One of the obstacles found is the fact that $75 \%$ of doctors never received a proposal to participate in the training and $50 \%$ are unaware that CFM offers the training to determine brain death. The responses to the questionnaire also showed that only half of the physicians who performed protocol opening completed the diagnosis, but there is also a doctor who, without training, declared to have closed the diagnosis of BD. CONCLUSION: CFM Resolution No. 2,173 / 2017 increases the credibility and confidence in Brazilian medicine, brings updated knowledge of a challenging diagnosis and has indirect impacts in the hope of saving more lives. After concluding the analysis of this study, the low qualification for $B D$ diagnosis carried by doctors is evident in the ICU scenario that presents significant deaths due to brain death. Dexterity in determining $M E$ is essential for organ donation, which is why it deserves its due relevance. In view of the above, it is expected that this study will encourage CFM to offer more training opportunities, and that medical professionals will seek to improve and update their knowledge to determine brain death according to the new rules.

Keywords: Brain death; Diagnosis; Intensive Care Units. 


\section{INTRODUÇÃO}

A concepção de Morte Encefálica é apresentada como um diagnóstico de forte impacto na sociedade e que gera muitos debates, principalmente pelo crescente avanço no sistema de transplante de órgãos. O presente estudo indaga se os médicos estão atualizando seus conhecimentos conforme a Resolução CFM n ${ }^{\circ}$ 2.173/2017, visto que o diagnóstico de morte encefálica agora é obrigatório para todos os casos suspeitos, não apenas para os potenciais doadores de órgãos. $O$ resultado do estudo brasileiro realizado em 2014 nas Unidades de Terapias Intensivas (UTIs) do estado de Teresina-PI, para avaliação de conhecimento de médicos intensivistas sobre morte encefálica, demostrou que apenas $20 \%$ possuem título de especialista em terapia intensiva e $37,8 \%$ responderam adequadamente o horário de óbito em doador de órgãos. Nesse estudo o conceito de morte encefálica foi definido corretamente pela maioria $(85,6 \%)$, todavia os intensivistas com menos de 10 anos de formação acertaram mais (96,8\%). Esses dados ratificam a necessidade de meios para colaborar e aperfeiçoar o conhecimento dos profissionais intensivistas ao diagnosticar morte encefálica.

Morte encefálica é definida como a cessação irreversível das funções corticais cerebrais e do tronco encefálico. No Brasil, é sinônimo de morte humana, pois indivíduos nessa situação são incapazes de recuperar o pleno controle de suas funções vitais. É condição essencial para a retirada post mortem de tecidos e órgãos humanos para transplantes, conforme regulamentado pela Lei 9.434/97, a qual também determina que seja de competência do Conselho Federal de Medicina (CFM) a definição de critérios clínicos e tecnológicos para o diagnóstico de morte encefálica. (MAGALHÃES et al., 2016, p.157).

As normas brasileiras para diagnóstico de morte encefálica (ME), de acordo com a Resolução CFM no 1.480/1997, são baseadas em critérios internacionais, apresentando alto grau de exatidão e confiança. A Resolução CFM nº 2.173/2017 traz maiores aprimoramentos na acurácia da determinação da morte encefálica, o ponto mais importante das novas normas é a exigência de capacitação dos médicos 
responsáveis por realização de testes clínicos e exames complementares. Diante dessa resolução, surge o questionamento de como está sendo executada a qualificação médica e se as normas estabelecidas pelo CFM estão sendo devidamente cumpridas após sua publicação.

De acordo com Shappel et al., revisou a determinação da ME em 226 doadores de órgãos de 68 hospitais dos Estados Unidos da América (EUA). Os profissionais que realizaram procedimentos foram intensivistas $(37 \%)$, neurologistas (28\%), neurocirurgiões (15\%) e de outras especialidades (20\%). (CONSELHO FEDERAL DE MEDICINA, 2017).

O presente estudo visa analisar o grau de habilitação dos médicos intensivistas, em vista da alta demanda e perícia necessárias para determinação de ME nas UTIs. Segundo Schein (2008, p.145), o atraso ou falha nessa etapa resulta em custos inapropriados, ocupação de um leito de UTI, aumento do sofrimento familiar e não oferta de órgãos para transplantes.

São considerados capacitados médicos com no mínimo um ano de experiência no atendimento de pacientes em coma, que tenham acompanhado ou realizado pelo menos dez determinações de ME e realizado treinamento específico para esse fim em programa que atenda as normas determinadas pelo Conselho Federal de Medicina. (CONSELHO FEDERAL DE MEDICINA, 2017).

O Brasil apresenta um dos maiores sistemas público de transplantes do mundo. Depois de diagnosticada a $\mathrm{ME}$, surge a possibilidade de captação de órgãos, que é reservado à família ou responsável a decisão de aceitar o transplante. No entanto, o passo inicial para se pensar em propiciar a doação de órgãos é ter uma equipe treinada e capacitada para diagnosticar ME com total destreza, para que não ocorra a dissipação dos potenciais doadores.

No Brasil, de acordo com o Registro Brasileiro de Transplantes, de 2014, foram notificados 9351 potenciais doadores, porém, apenas 2713 doações foram efetivadas, tendo em vista que havia 28969 pacientes ativos na lista de espera até o final do ano em questão. (SILVA et al., 2018, p. 52). 
A aplicação de questionário aos médicos das unidades de terapia intensiva permite transparecer se os profissionais capacitados, conforme o CFM, estão realizando o diagnóstico. Permite também questionar como o Conselho Federal de Medicina está disponibilizando o curso de capacitação após o lançamento da nova resolução em 2017. Os benefícios de um laudo correto vão além do diagnóstico, a sociedade evolui no processo de possibilitar a doação de órgãos e presenteando outras vidas a partir disso.

\section{METODOLOGIA}

Trata-se de estudo transversal e quantitativo que será realizado por levantamento de dados a partir de um questionário. Pesquisa realizada nos municípios de Cajazeiras e Sousa, situados no alto sertão paraibano do estado da Paraíba. Cidades que se destacam por atender a mais de 42 municípios paraibanos da região, além de municípios de estados vizinhos, como Pernambuco, Rio Grande do Norte e Ceará. Especificamente, a pesquisa foi facilitada pelo aplicativo de mensagem WhatsApp e o objeto de estudo são os médicos plantonistas do Hospital Regional de Sousa e Hospital Regional de Cajazeiras no setor da UTI.

A população abordada no estudo é definida por todos os médicos plantonistas da Unidade de Terapia Intensiva, independentemente de sua especialidade. Estiveram sujeitos à pesquisa todos os profissionais com bacharelado em medicina. A amostra é composta por 16 médicos plantonistas, referente ao (s) plantonista (s) de cada dia, por 15 dias, durante o período de 24 de março até dia 07 de abril de 2021.

Foram vinculados todos os médicos Plantonistas que atuam no Hospital Regional de Sousa (HRS) e Hospital Regional de Cajazeiras (HRC) no setor da UTI. E que se dispuseram a participar da pesquisa. Os excluídos da pesquisa foram os médicos não plantonistas da UTI do HRS e HRC.

A presente pesquisa foi executada através de dados primários, prédeterminados pelo pesquisador através de um questionário formulado com respostas 
prontas, autoaplicável, respondido por formulário, após reconhecimento de termo de consentimento, o qual será aplicado aos médicos plantonistas da UTI do HRC e HRS da escala da última semana de março e primeira semana de abril. O questionário foi disponibilizado através de um link, via WhatsApp, gerado pelo Google Forms e no próprio formulário já consta o Termo de Consentimento Livre e Esclarecido (TCLE).

A coleta ocorreu por meio de questionário, disponibilizado através do link gerado pelo Google Forms e enviado via WhatsApp. O contato dos plantonistas foi adquirido com a Direção Técnica de cada hospital.

Os dados foram armazenados em uma planilha eletrônica do programa Microsoft Office Excel 2010 e, posteriormente, transferidos para a tabela do programa Statistical Packagefor the Social Sciences (SPSS) versão 22.0, analisados mediante a literatura, com auxílio de estatística descritiva simples e exploratória, indicado para estudos nos quais se pretende quantificar e descrever os resultados, por meio do programa sem modificá-los.

O projeto foi encaminhado ao Comitê de Ética em Pesquisa da Faculdade Santa Maria seguindo os padrões éticos determinados na Resolução $n^{\circ} 466 / 12$, do Conselho Nacional de Saúde (CNS), que regulamenta a pesquisa em seres humanos, assegurando aos participantes informações sobre os objetivos, aceitação e desistência da participação em qualquer momento, sem nenhum prejuízo para o mesmo. Após a apreciação favorável do Comitê de Ética em Pesquisa (CEP), foi iniciada a pesquisa de campo.

\section{RESULTADOS}

As direções técnicas do HRC e HRS forneceram o contato de 16 médicos plantonistas da UTI, foram contatados via WhatsApp e enviado o link para preenchimento do questionário por meio de formulário. Apenas oito profissionais se disponibilizaram a responder, os demais não justificaram o desejo de não participar. 
O perfil dos médicos entrevistados está apresentado na tabela 1 e gráficos 1 e 2 . Todos atuam em UTI de adultos.

Tabela 1. Caracterização do perfil profissional dos médicos plantonistas da UTI. Cajazeiras, PB, 2021.

\begin{tabular}{|lll|}
\hline SEXO? & TEMPO DE FORMADO? & $\begin{array}{l}\text { QUAL } \\
\text { ESPECIALISTA POSSUI? }\end{array}$ \\
\hline Masculino $(75 \%)$ & Menos de 5 anos $(25 \%)$ & Medicina Intensiva $(25 \%)$ \\
\hline Feminino $(25 \%)$ & Entre 5 e 10 anos $(50 \%)$ & Nefrologia $(12,5 \%)$ \\
\hline & Mais de 10 anos $(25 \%)$ & Nenhum $(62,5 \%)$ \\
\hline
\end{tabular}

Fonte: Dados da pesquisa, 2021.

Gráfico 1. Taxa de médicos especialistas. Cajazeiras, PB, 2021.

\section{POSSUI ESPECIALIDADE MÉDICA?}

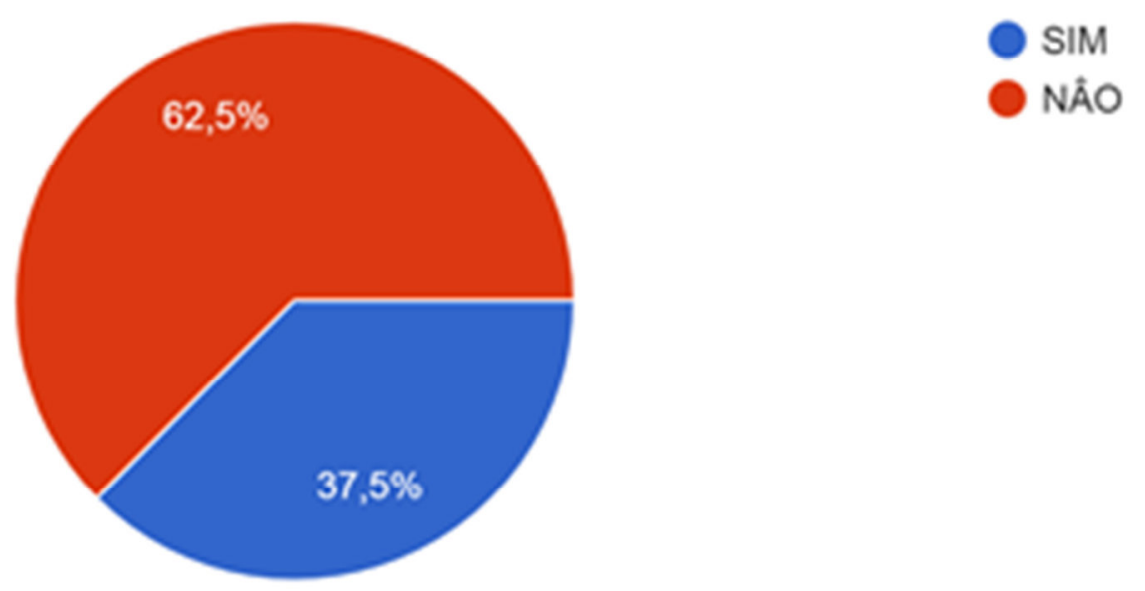

Fonte: Dados da pesquisa, 2021. 
Gráfico 2. Idade dos participantes em anos. Cajazeiras, PB, 2021.

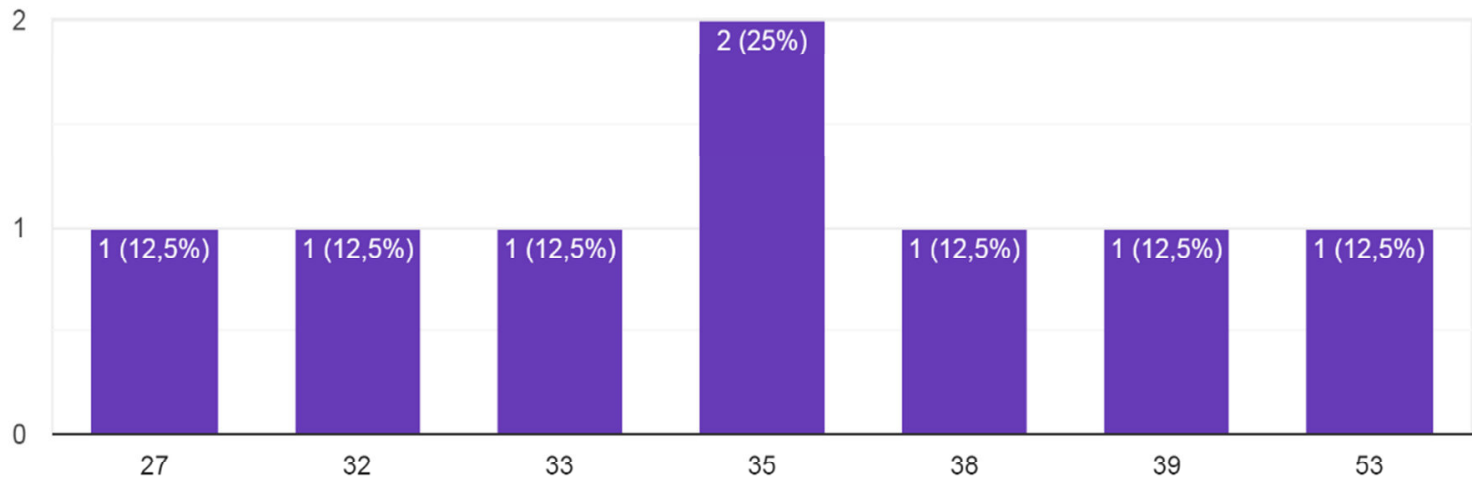

Fonte: Dados da pesquisa, 2021.

Observou-se predomínio de profissionais com menos de dez anos de exercício da medicina (75\%). A maioria dos participantes $(62,5 \%)$ também não cursou residência médica. Dentre os que apresentam especialização, temos em medicina intensiva (25\%) e em nefrologia (12,5\%).

Dos participantes entrevistados, a grande maioria não tem residência médica em Medicina Intensiva, ratificando as dificuldades de encontrar especialistas em manejo de paciente grave no interior paraibano. Há $25 \%$ de médicos com menos de 5 anos de exercício da profissão e sem qualquer especialidade médica, denotando um cenário de médicos com pouca experiência.

Tabela 2. A atuação profissional dos médicos participantes. Cajazeiras, PB, 2021.

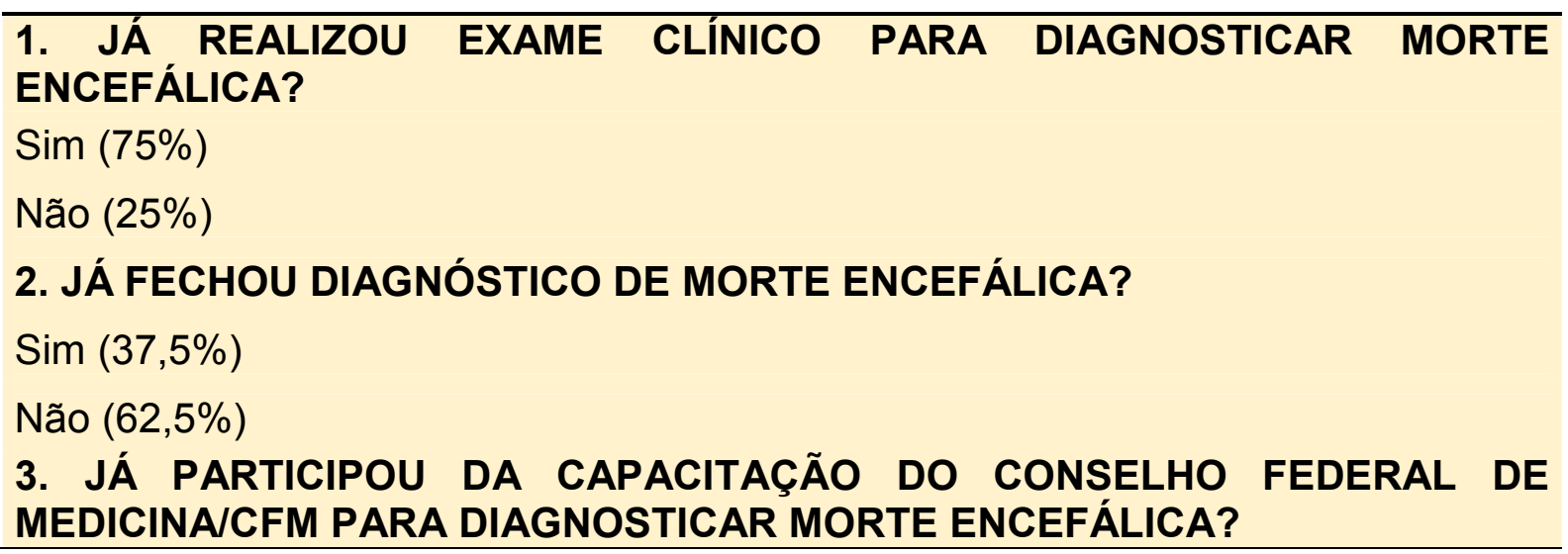


$\operatorname{Sim}(25 \%)$

Não $(75 \%)$

4. TEM CONHECIMENTO QUE O CFM OFERTA O CURSO DE CAPACITAÇÃO PARA ME?

$\operatorname{Sim}(50 \%)$

Não $(50 \%)$

5. JÁ LHE FOI OFERTADO A CAPACITAÇÃO PARA DETERMINAÇÃO DE MORTE ENCEFÁLICA EXIGIDA PELO CFM?

$\operatorname{Sim}(25 \%)$

Não $(75 \%)$

6. APÓS TESTE CLÍNICO PARA ME VOCÊ UTILIZA EXAMES COMPLEMENTARES?

$\operatorname{Sim}(75 \%)$

Não $(25 \%)$

Fonte: Dados da pesquisa, 2021.

Tabela 3. Exames complementares. Cajazeiras, PB, 2021.

\section{PRINCIPAIS EXAMES COMPLEMENTARES UTILIZADOS:}

Eletroencefalograma (50\%)

Angiotomografia Cerebral (12,5\%)

Ultrassom-Doppler Transcraniano (12,5\%)

Nenhum (25\%)

Fonte: Dados da pesquisa, 2021.

A quantidade de médicos que realizaram abertura de protocolo (75\%) é maior que a quantidade de médicos que já fecharam diagnóstico (37,5\%) de ME, ou seja, a metade dos que iniciaram os testes, logo demonstra que há insegurança ou mesmo dificuldades para conseguir êxito. Como esperado, a imensa maioria não está apta a determinar ME conforme a nova resolução do CFM, visto que $62,5 \%$ dos participantes nunca fecharam diagnóstico de ME e apenas três profissionais $(37,5 \%)$ estão aptos conforme nova resolução. Dentre os autorizados a determinar ME temos um participante não especialista que recebeu treinamento e dois médicos com residência em medicina intensiva, embora estes necessitem atualizar os conhecimentos pois não participaram da capacitação. Há um médico especialista e 
apto que nunca fechou diagnóstico de ME, como também foi identificado um médico não habilitado que já concluiu o diagnóstico, logo, coloca-se em questão a experiência e ética profissional para assumir tamanha responsabilidade.

A grande maioria dos médicos (75\%) não receberam proposta para participar da capacitação e $50 \%$ desconhece que o CFM oferece a capacitação para determinar morte encefálica. Grande parte dos médicos desta pesquisa realiza exame clínico para ME, mas não prossegue no fechamento de protocolo. Tais fatos geram prejuízos para o SUS, tanto pela ocupação desnecessária de leitos hospitalares, bem como o não encaminhamento do paciente para captação de órgãos, caso seja potencial doador.

É importante destacar que $75 \%$ dos médicos participantes citaram corretamente exames complementares obrigatórios para fechar protocolo de ME, os demais omitiram. Dentre os exames mais citados temos o eletroencefalograma.

\section{DISCUSSÃO}

A pesquisa tem o intuito de observar avanços por parte da equipe médica em conduzir pacientes com morte encefálica de acordo com a Resolução CFM $n^{\circ}$ 2.173/2017, e se o CFM está promovendo os objetivos da resolução. A resolução estabelece, de forma clara, a obrigatoriedade de fechar o diagnóstico de ME mesmo que o paciente não seja candidato a transplante de órgãos. Essa nova resolução ratifica o histórico do Brasil de segurança próxima a 100\% no protocolo para diagnóstico de morte encefálica, desde que seja devidamente e cautelosamente realizado. Porém, uma das falhas existente no cenário brasileiro é a omissão em prosseguir a determinação de morte encefálica. Isso é consequência da não capacitação da maioria dos profissionais médicos plantonista de UTI, sendo optado por constar outras causas base na Declaração de Óbito.

As dificuldades que o CFM encontra para oferecer a capacitação é um dos vieses a ser destacado. A Resolução deixa clara a necessidade de capacitação médica, mas a entidade não planejou tais cursos para abranger e treinar os médicos 
que trabalham com pacientes aptos a receber diagnóstico de ME. Como exposto na análise da pesquisa, o CFM não tem gerenciado o oferecimento de cursos de capacitação teórico-prático, além disso, a fiscalização do cumprimento da nova resolução não é colocada em prática.

A análise do perfil dos participantes e as respostas nesta pesquisa esclarecem a realidade presente no Alto Sertão paraibano. Fica explícito e questionável a dificuldade em determinar morte encefálica devido a não preparação dos $62,5 \%$ dos médicos plantonistas de UTI participantes dessa pesquisa. Também foi possível observar que as UTIs dispõem de poucos médicos plantonistas especialista em Medicina Intensiva, ou até mesmo conta com médicos sem especialização e com pouca experiência em lidar com pacientes graves.

Também há outros vieses a ser destacado, por exemplo as dificuldades para realização de exames complementares e recursos limitados para manutenção dos parâmetros adequados do paciente exigido pelo protocolo. Essas compreendem algumas dificuldades para fechar diagnóstico.

A disponibilidade de recursos, médicos especialistas e acessível deslocamento são vantagens grotescas presentes em grandes centros e capitais, tornando mais viável o processo de transplante de órgãos. O presente estudo atenta para a situação dos pacientes com suspeita de morte encefálica em UTIs do interior paraibano, cujo manejo adequado desses pacientes é uma realidade distante. Um dos grandes impasses, ou mesmo prejuízos para alimentar a rede de transplante de órgãos, é a dificuldade na descentralização, no entanto, um dos tripés essenciais é a participação de profissionais capacitados desde a suspeição de morte encefálica. Dentre as vantagens de um diagnóstico conduzido corretamente temos: dar respaldo à família, a fim de diminuir aflição, diminuir custos hospitalares, assim como evitar a ocupação de leito por paciente sem prognóstico e, principalmente, fomentar o sistema de transplante de órgãos. 


\section{CONCLUSÃO}

A relevância da Resolução CFM n 2.173/2017 aumenta a credibilidade e confiança na medicina brasileira, traz atualização de conhecimentos de um diagnóstico desafiador e que tem impactos indiretos na esperança de salvar mais vidas. A finalidade deste estudo não é sondar a eficiência e a capacidade médica, ou seja, não teve intuito de testar o conhecimento médico para criticar sua imperícia, visto que os médicos trabalham com recursos e oportunidades limitadas. Trata-se de uma análise da habilitação portada pelo médico diante de um cenário de UTI que apresenta significativos óbitos por ME.

Ressalva-se a responsabilidade inerente ao CFM como aos Conselhos Regionais de Medicina em propiciar uma capacitação continuada dos médicos para abordar com total perícia um paciente com suspeita de ME. Perante a carência de médicos intensivistas em UTI é indispensável oferecer e, por conseguinte, exigir que se tenha profissionais altamente preparados para situações de morte encefálica.

Além de ser imprescindível para a doação de órgãos de pessoas falecidas, o diagnóstico eficaz de morte encefálica exerce influência na ética profissional, garantindo maior assistência a pacientes e familiares, mas também contribuindo para ocupação racional dos leitos de UTI, evitando uso de forma desnecessária. Assim, é fundamental que o médico intensivista domine a determinação de morte encefálica, seja capaz de identificá-la e abordá-la de acordo com as novas normas médicas e legais vigentes no Brasil.

Seria de extrema organização a criação de um banco de dados que verificasse a compatibilidade de uma Declaração de Óbito com o grau de perícia do médico responsável. Todavia, cabe ao CFM oferecer mais suporte e exigências de financiamento das esferas públicas, além de descentralizar os centros de referência em transplante de órgãos. Diante do apresentado, espera-se que este estudo instigue o CFM a oferecer mais oportunidades de capacitação e que os profissionais médicos busquem um melhor aperfeiçoamento e atualização dos seus conhecimentos para determinar morte encefálica. 


\section{REFERÊNCIAS BIBLIOGRÁFICAS}

CATARINO, M. M. Parecer CRM-MG No 133/2018 - PROCESSO-CONSULTA No 6.178/2017. Conselho Regional de Medicina - MG. Belo Horizonte, 28 de setembro de 2018.

Conselho Federal de Medicina (Brasil). Resolução $n^{\circ}$ 2.173, de 23 de novembro de 2017. Estabelece os critérios para determinação de ME. Diário Oficial da União. Seção I, p. 274-6, 15 de dezembro de 2017.

Conselho Federal de Medicina (Brasil). Resolução $n^{\circ}$ 1.480, de 8 de agosto de 1997. Estabelece os critérios para caracterização de ME. Diário Oficial da União. 21 ago 1997.

ESCUDERO, D et al. Intensive care practices in brain death diagnosis and organ donation. The Association of Anaesthetists of Great Britain and Ireland, v. 70, p. 1130-1139, 2015.

MAGALHÃES, J.V. et al. Avaliação do conhecimento de médicos intensivistas de Teresina sobre morte encefálica. Rev. bioét. (Impr.). v. 24, n. 1, p. 156-64, 2016.

ORBAN, J.C.; FERRET, E.; JAMBOU, P.; ICHAI C. Confirmation of brain death diagnosis: A study on French practice. Socie' te' franc, aise d'anesthe' sie et de re'animation, Anaesth Crit Care Pain Med. v. 34, p. 145-150, 2015.

SCHEI, A.E. et al. Avaliação do conhecimento de intensivistas sobre morte encefálica. Revista Brasileira de Terapia Intensiva, v. 20, n. 2, Abril/Junho, 2008.

SILVA, F.A.A.; CUNHA, D.S.P.; LIRA J.A.C. et al. Morte encefálica e manutenção de órgãos: conhecimento dos profissionais intensivistas. Rev enferm UFPE on line, v. 12, n. 1, p. 51-8, 2018.

WAHLSTER, S. et al. Brain death declaration. American Academy of Neurology, v. 84, May 5, 2015. 